\title{
XLV. On the kinetic interpretation of the dissipation function
}

\section{Dr. Ladislas Natanson}

To cite this article: Dr. Ladislas Natanson (1895) XLV. On the kinetic interpretation of the dissipation function, Philosophical Magazine Series 5, 39:240, 455-460, DOI: $10.1080 / 14786449508620743$

To link to this article: http://dx.doi.org/10.1080/14786449508620743

曲 Published online: 08 May 2009.

Submit your article to this journal $\lceil\pi$

Џ Article views: 4

Q View related articles $\asymp$ 
Between each grain and its neighbours there is a true surface, the tension of which probably depends in some measure upon the relationship of their axes to each other.

Now we know that a by no means unimportant portion of the energy of a solid body depends upon its surface area ; and I would suggest also that it depends in some measure upon the surfaces separating the crystal units, of which it is composed, from each other, even when they are of similar composition.

In a block of glacier-ice, therefore, or in a block of granite, the total energy depends, not only upon its temperature and volume, but also upon the energy of the surface-tensions of its component grains, whether they be similar or dissimilar in composition.

Now it is an important theorem of Dynamies that, for the stable equilibrium of a system, the potential energy of the whole must be a minimum. For instance, when immiscible liquids are intimately mixed, work is done in increasing their surface-energy, and the energy thus rendered potential serves to again separate them. Glacier-ice is in much the same condition. Its potential energy is always tending to a minimum, a condition which can only be reached by the disappearance of the interfaces between the crystal-grains. Being viscous, it is unable to do more than delay the disappearance of the interfaces, and the grains would continue to grow larger and larger with the lapse of time were it not for the fact that they are broken in the production of the ribboned or veined structure.

For the same reason masses of all liquids possessing structure, whatever their viscosities may be, eventually become single crystalline particles.

R. M. Deeley.

XLV. On the Kinetic Interpretation of the Dissipation Function. By Dr. Ladislas Natanson, Professor of Natural Philosophy, University of Cracow*.

1. $\mathrm{N}$ the following paper we consider a fluid mediun from Maxwell's point of view (see the paper "On the Dynamical Theory of Gases," 'Scientific Papers,' ii. 26) ; we suppose it to be composed of a great number of moving molecules. Let $\xi, \eta, \zeta$ be the components of the individual (or "molecular") velocity of a molecule; and let $u, v, w$ be

* Translated from " $R$ szprawy" (Transactions) of the Cracow Academy of Sciences, Math. and Phys. Section, vol, xxix. Communicated by the Author. 
the components of the mean (or "molar") velocity within an element $d x d y d z$ of volume; $n d x d y d z$ may represent the number of molecules within that element. The mass of a molecule being $\mathrm{M}, \rho=\mathrm{M} n$ being the density of the medium, the kinetic energy of a molecule is

$$
\frac{1}{2} \mathrm{M}\left\{(u+\xi)^{2}+(v+\eta)^{2}+(w+\zeta)^{2}\right\} ; . . .
$$

and the total kinetic energy of a portion of the medium consists of the two following parts :-(1) the kinetic energy of the visible motion,

$$
\mathrm{K}=\frac{1}{2} \iiint \rho\left(u^{2}+v^{2}+w^{2}\right) d x d y d z ; . . .
$$

it is this energy that, in Hydrodynamics, is taken into account ; (2) the molecular or heat-energy,

$$
\mathrm{E}=\frac{1}{2} \iiint \rho\left(\overline{\xi^{2}}+\overline{\eta^{2}}+\overline{\zeta^{2}}\right) d x d y d z . \quad . \quad . \quad .
$$

In these expressions the integrations are supposed to be performed throughout the volume occupied by the medium; and $\overline{\xi^{2}}$ and similar symbols represent the mean values of $\xi^{2}$ \&c. for the molecules within an element. We have

$$
\bar{\xi}=0 ; \quad \bar{\eta}=0 ; \quad \bar{\zeta}=0
$$

At the point $(x, y, z)$ we have the normal component pressures,

$$
p_{x x}=\rho \overline{\xi^{2}} ; \quad p_{y y}=\rho \overline{\eta^{2}} ; \quad p_{z z}=\rho \overline{\zeta^{2}} ; . . .
$$

and the tangential,

$$
p_{y z}=p_{z y}=\rho \overline{\eta \zeta} ; \quad p_{z x}=p_{x z}=\rho \overline{\zeta \xi} ; \quad p_{x y}=p_{y z}=\rho \bar{\xi} \bar{\eta} .
$$

Let $\mathrm{Q}$ be any property of the molecule which can be expressed as a function of $(u+\xi),(v+\eta)$, and $(w+\xi)$. Then writing $d / d t$ for the actual or total variation of $\bar{Q}$, and $\delta / \delta t$ for any alteration of $\bar{Q}$ that can be due to the mutual interference of molecules,

$$
\begin{aligned}
& \left.\rho \frac{d \overline{\mathrm{Q}}}{d t}+\frac{\partial}{\partial x} \overline{(\bar{\xi}} \rho\right)+\frac{\partial}{\partial y}(\overline{\eta \mathrm{Q}} \rho)+\frac{\partial}{\partial z}(\overline{\zeta \mathrm{Q}} \rho) \\
& \quad=\rho \frac{\delta \overline{\mathrm{Q}}}{\delta t}+\rho \mathrm{X} \frac{\partial \overline{\mathrm{Q}}}{\partial u}+\rho \mathrm{Y} \frac{\partial \overline{\mathrm{Q}}}{\partial v}+\rho \mathrm{Z} \frac{\partial \overline{\mathrm{Q}}}{\partial w} ; .
\end{aligned}
$$

$\mathrm{X}, \mathrm{Y}, \mathrm{Z}$ denote here the components, at $(x, y, z)$, of acceleration due to external forces. From this fundamental equation (7) the equation of continuity, as well as the equations of motion,

$$
\rho \frac{d u}{d t}+\frac{\partial}{\partial x}\left(\rho \overline{\xi^{2}}\right)+\frac{\partial}{\partial y}(\rho \overline{\eta \xi})+\frac{\partial}{\partial z}(\rho \overline{\zeta \xi})=\rho \mathrm{X}, \ldots
$$

immediately follow. Let us now put

$$
Q=(u+\xi)^{2}+(v+\eta)^{2}+(w+\zeta)^{2} ; \quad \text {. . . }
$$


we obtain

$$
\begin{aligned}
& \rho \frac{d}{d t}\left(u^{2}+v^{2}+w^{2}+\overline{\xi^{2}}+\overline{\eta^{2}}+\overline{\zeta^{2}}\right) \\
& \quad+\frac{\partial}{\partial x}\left(2 u \rho \overline{\xi^{2}}+2 v \rho \overline{\xi \eta}+2 w \rho \overline{\xi \zeta}+\rho \overline{\xi^{3}}+\rho \overline{\xi \overline{\eta^{2}}}+\rho \overline{\xi \zeta^{2}}\right) \\
& \quad+\frac{\partial}{\partial y}\left(2 u \rho \overline{\eta \xi}+2 v \rho \overline{\eta^{2}}+2 w \rho \overline{\eta \zeta}+\rho \overline{\eta \xi^{2}}+\rho \overline{\eta^{3}}+\rho \overline{\eta \overline{\zeta^{2}}}\right) \\
& \quad+\frac{\partial}{\partial z}\left(2 u \rho \overline{\zeta \xi}+2 v \rho \bar{\zeta}+2 w \rho \overline{\zeta^{2}}+\rho \overline{\zeta \xi^{2}}+\rho \overline{\zeta \eta^{2}}+\rho \overline{\zeta^{3}}\right) \\
& \quad=2 \rho(u \mathrm{X}+v \mathrm{Y}+w \mathrm{Z})+\rho \frac{\delta}{\delta t}\left(u^{2}+v^{2}+w^{2}+\overline{\xi^{2}}+\overline{\eta^{2}}+\overline{\zeta^{2}}\right) .
\end{aligned}
$$

By (8) this equation is reduced to

$$
\begin{aligned}
\frac{1}{2} & \rho \frac{d}{d t}\left(\overline{\xi^{2}}+\overline{\eta^{2}}+\overline{\zeta^{2}}\right)+\rho \overline{\xi^{2}} \frac{\partial u}{\partial x}+\rho \overline{\eta^{2}} \frac{\partial v}{\partial y}+\rho \overline{\zeta^{2}} \frac{\partial w}{\partial z} \\
& +\rho \overline{\eta \zeta}\left(\frac{\partial v}{\partial z}+\frac{\partial w}{\partial y}\right)+\rho \overline{\zeta \xi}\left(\frac{\partial w}{\partial x}+\frac{\partial u}{\partial z}\right)+\rho \overline{\xi \eta}\left(\frac{\partial u}{\partial y}+\frac{\partial v}{\partial x}\right) \\
& +\frac{1}{2} \frac{\partial}{\partial x}\left(\rho \overline{\xi^{3}}+\rho \overline{\xi \eta^{2}}+\rho \overline{\xi^{2}}\right)+\frac{1}{2} \frac{\partial}{\partial y}\left(\rho \overline{\eta^{2}}+\rho \overline{\eta^{3}}+\rho \overline{\eta \zeta^{2}}\right) \\
& +\frac{1}{2} \frac{\partial}{\partial z}\left(\rho \overline{\xi^{2}}+\rho \overline{\eta^{2}}+\rho \overline{\zeta^{3}}\right) \\
& =\frac{1}{2} \rho \frac{\delta}{\delta t}\left(u^{2}+v^{2}+w^{2}+\overline{\xi^{2}}+\overline{\eta^{2}}+\overline{\zeta^{2}}\right) . . . . . . .
\end{aligned}
$$

$\Lambda$ ll these equations are quite independent of any view we mav entertain as to the intimate nature of molecules and as to the law of force between them ; accordingly they may be said to belong to the Kinematical Part of the Theory of constitution of fluids.

2. If we suppose that the molecules of the fluid do not contain any internal energy (or that they contain such energy in absolutely constant amount) the energy of a molecule will be only that which depends on the velocity of its centre of inertia. Assuming this equation (11) may be transformed. Put for brevity,

$$
\begin{aligned}
\frac{\partial u}{\partial x}=a ; \quad \frac{\partial v}{\partial y}=b ; \quad \frac{\partial w}{\partial z}=c ; . . . \\
\frac{\partial v}{\partial z}+\frac{\partial w}{\partial y}=\mathrm{A} ; \quad \frac{\partial w}{\partial x}+\frac{\partial u}{\partial z}=\mathrm{B} ; \quad \frac{\partial u}{\partial y}+\frac{\partial v}{\partial v}=\mathrm{C} ;
\end{aligned}
$$

and let us add to the left-hand side

$$
\frac{1}{2}\left(\overline{\xi^{2}}+\overline{\eta^{2}}+\overline{\zeta^{2}}\right) \frac{d \rho}{d t}+\frac{1}{2} \rho\left(\overline{\bar{\xi}^{2}}+\overline{\eta^{2}}+\overline{\zeta^{2}}\right) \theta, \quad . \quad .
$$

where $\theta=a+b+c$. Multiplying by $d x d y d z$ and integrating 
throughout the volume occupied by the medium, we have then

$$
\begin{aligned}
& \frac{\partial \mathrm{E}}{\partial t}+\frac{1}{2} \iint \rho\left(\overline{\xi^{2}}+\overline{\eta^{2}}+\overline{\zeta^{2}}\right)(l u+m v+n w) d \mathrm{~S} \\
+ & \iiint\left[\rho \bar{\xi}^{2} a+\rho \bar{\eta}^{2} b+\rho \bar{\zeta}^{2} c+\rho \overline{\eta \zeta} \mathrm{A}+\rho \overline{\zeta \xi} \mathrm{B}+\rho \overline{\xi \eta} \mathrm{C}\right] d x d y d z=0 ;
\end{aligned}
$$

here the direction-cosines of the normal to the element $d \mathrm{~S}$ of the surface are denoted by $l, m, n$. If the medium cannot flow across the surface, the second term on the left-hand side may be omitted. Assuming this and calculating $\partial \mathrm{K} / \partial t$ from equations (8) in a similar way we find

$\frac{\partial \mathrm{K}}{\partial t}-\iiint\left[\rho \bar{\xi}^{2} a+\rho \overline{\eta^{2}} b+\rho \bar{\zeta}^{2} c+\rho \overline{\eta \xi} \mathrm{A}+\rho \overline{\xi \xi} \mathrm{B}+\rho \bar{\xi} \eta \mathrm{C}\right] d x d y d z$

hence

$$
=\iiint \rho(u \mathrm{X}+v \mathrm{Y}+w \mathrm{Z}) d x d y d x ; .
$$

$$
\frac{\partial \mathrm{K}}{\partial t}=-\frac{\partial \mathrm{E}}{\partial t}+\iiint \rho(u \mathrm{X}+v \mathrm{Y}+w \mathrm{Z}) d x d y d z, \ldots
$$

i.e. molar energy is subject to change from two sources, the first being the influence of external forces, the second being the possible transformation of molecular energy $\mathrm{E}$ into molar energy $\mathrm{K}$ or vice vers $\hat{a}$. Let us denote the rate of change of $K$ due to the second source of variation by $\partial^{\prime} \mathrm{K} / \partial t$; we have

$$
\frac{\partial \mathrm{E}}{\partial t}=-\frac{\partial ' \mathrm{~K}}{\partial t}=-\iiint\left[\rho \overline{\xi^{2}} a+\rho \overline{\eta^{2}} b+\rho \bar{\zeta}^{2} c+\rho \bar{\eta} \zeta \mathrm{A}+\rho \overline{\zeta \xi \mathrm{B}}+\rho \overline{\xi \eta} \mathrm{C}\right] d x d y d z .
$$

Let us put

$$
\begin{aligned}
& 3 p=\rho \overline{\xi^{2}}+\rho \overline{\eta^{2}}+\rho \zeta^{\overline{2}} . \quad . \quad . \quad . \quad . \quad .(19) \\
& \mathrm{F}=\left(p-\rho \overline{\xi^{2}}\right) a+\left(p-\rho \overline{\eta^{2}}\right) b+\left(p-\rho \overline{\zeta^{2}}\right) c-\rho \bar{\zeta} \overline{\mathrm{A}}-\rho \overline{\zeta \bar{\xi}} \mathrm{B}-\rho \overline{\xi \eta} \mathrm{C} \\
& =-\rho \overline{\bar{\zeta}^{2}}\left(a-\frac{1}{3} \theta\right)-\rho \overline{\eta^{2}}\left(b-\frac{1}{3} \theta\right)-\overline{\rho \zeta^{2}}\left(c-\frac{1}{3} \theta\right)-\rho \bar{\eta} \overline{\mathrm{A}}-\rho \overline{\zeta \xi} \mathrm{B}-\rho \overline{\xi \eta} \mathrm{C} \\
& =\left(p-\rho \bar{\xi}^{2}\right)\left(a-\frac{1}{3} \theta\right)+\left(p-\rho \overline{\eta^{2}}\right)\left(b-\frac{1}{3} \theta\right)+\left(p-\rho \overline{\zeta^{2}}\right)\left(c-\frac{1}{3} \theta\right) \\
& -\rho \bar{\eta} \overline{\mathrm{A}}-\rho \overline{\zeta \xi} \mathrm{B}-\rho \overline{\xi \eta} \mathrm{C} \text {. }
\end{aligned}
$$

Equation (18) may then be written

$$
\frac{\partial \mathrm{E}}{\partial t}=-\frac{\partial^{\prime} \mathrm{K}}{\partial t}=\iiint(\mathrm{F}-p \theta) d x d y d z ; \quad .
$$

the molecular energy $\mathrm{E}$ is therefore subject to change from two sources : first, the work of the ordinary average prossure, and second, the effect of a disturbance giving rise to tangential pressures and to inequality of the normal ones, $F$ being the rate (per unit of volume and time) at which molecular energy is generated by the effect of the disturbance.

Put in (7)

$$
\mathrm{Q}=(u+\xi)^{2} ; \quad \mathrm{Q}=(v+\eta)(w+\xi) ; \quad .
$$


if the disturbance is not very violent, we shall find

$$
\begin{aligned}
\frac{\delta}{\delta t}\left(p-\rho \overline{\xi^{2}}\right) & =-2 p\left(a-\frac{1}{3} \theta\right) . \quad . \quad . \quad . \\
\frac{\delta}{\delta t}(\rho \overline{\eta \bar{\zeta}}) & =p \mathrm{~A}, \quad . \quad . \quad . \quad . \quad .
\end{aligned}
$$

and other equations which may be written down from symmetry. Hence

$$
\begin{aligned}
\mathrm{F}=-\frac{1}{4 p} \frac{\delta}{\delta t}\left\{\left(p-\rho \overline{\xi^{2}}\right)^{2}+\left(p-\rho \overline{\eta^{2}}\right)^{2}+\left(p-\rho \overline{\zeta^{2}}\right)^{2}\right. \\
\left.+2(\rho \overline{\bar{\zeta}})^{2}+2(\rho \overline{\zeta \xi})^{2}+2(\rho \overline{\xi \eta})^{2}\right\} . .
\end{aligned}
$$

From this it appears that the direction of that trausformation of energy the rate of which is $\mathrm{F}$ depends on the nature of the mutual action between molecules. Molar energy will become converted into molecular energy if molecular interaction is such as to tend to diminish the absolute values of the terms $p-\rho \xi^{2}, \rho \overline{\zeta \zeta}$ and similar terms; i. e. such as to tend gradually to calm the disturbance existing : $\mathrm{F}$ is then always a positive quantity and the opposite transformation of molecular energy into molar energy is impossible. That this is precisely what is realised in all fluids in Nature, as attested by the phenomenon of viscosity, cannot, however, be deduced from Kinematical Theory. We have ascertained, as it were, the path of change of the molar energy, but we are unable to say why one of the two possible directions of change is alway's selected.

If now we suppose the following equations to be given :-

$$
\begin{aligned}
p-\rho \overline{\xi^{2}} & =2 \mu\left(a-\frac{1}{3} \theta\right), . . \\
\rho \eta \bar{\zeta} & =-\mu \mathrm{A}, \quad . \quad . \quad .
\end{aligned}
$$

(the symbol $\mu$ denoting a constant, the coefficient of viscosity), and four other equations, to be written down from symmetry, we shall find it possible to complete our solution. Maxwell deduced these equations from his well-known assumption as to the law of force between molecules; Poisson, Sir G. G. Stokes, and others have given them in the ordinary Theory of Viscosity. It follows, then, that

$$
\begin{aligned}
\mathbf{F} & =\frac{1}{\mu}\left\{\frac{1}{2}\left(p-\rho \overline{\xi^{2}}\right)^{2}+\frac{1}{2}\left(p-\rho \overline{\eta^{2}}\right)^{2}+\frac{1}{2}\left({ }_{1} p-\rho \overline{\zeta^{2}}\right)^{2}+(\rho \overline{\eta \zeta})^{2}+(\rho \overline{\zeta \xi})^{2}+(\rho \overline{\xi \eta})^{2}\right\}, \\
& =\mu\left\{2\left(a-\frac{1}{3} \theta\right)^{2}+2\left(b-\frac{1}{3} \theta\right)^{2}+2\left(c-\frac{1}{3} \theta\right)^{2}+A^{2}+B^{2}+C^{2}\right\} \\
& =\frac{1}{\mu}\left\{\frac{1}{6}\left(\rho \overline{\eta^{2}}-\rho \overline{\zeta^{2}}\right)^{2}+\frac{1}{6}\left(\rho \overline{\zeta^{2}}-\rho \overline{\bar{\xi}^{2}}\right)^{2}+\frac{1}{6}\left(\rho \overline{\xi^{2}}-\rho \overline{\eta^{2}}\right)^{2}+(\rho \overline{\eta \zeta})^{2}+(\rho \overline{\zeta \xi})^{2}+(\rho \overline{\xi \eta})^{2}\right\} \\
& =\mu\left\{2(b-c)^{2}+\frac{2}{3}(c-a)^{2}+\frac{2}{3}(a-b)^{2}+A^{2}+B^{2}+C^{2}\right\},
\end{aligned}
$$


and thus $\mathrm{F}$ can never assume negative values. Also

$$
\mathrm{F}=\mu\left(2 a^{2}+2 b^{2}+2 c^{2}-\frac{2}{3} \theta^{2}+\mathrm{A}^{2}+\mathrm{B}^{2}+\mathrm{C}^{2}\right), \text {. }
$$

this is the expression for the "Dissipation Function" F originally given by Lord Rayleigh. The cause for this denomination is obvious. Suppose $a, b, c, \mathrm{~A}, \mathrm{~B}, \mathrm{C}$ to have their sign changed; the sign of $p \theta$ will change but that of $F$ will not; hence the term $p \theta$ corresponds to a reversible, the term $F$ to an irreversible phenomenon. Indeed the conversion of molar energy into molecular energy which goes on, of whatever kind the existing disturbance may be, is irreversible. We have thus before us an example of Dissipation of Energy in a purely dynamical system.

3 . If we now suppose that every molecule possesses an amount $\mathrm{M} h$ of internal energy in addition to that which depends on its motion of translation, we shall have

$$
\frac{\delta}{\delta t}\left[\frac{1}{2} \rho\left(u^{2}+v^{2}+w^{2}+\overline{\xi^{2}}+\eta^{\overline{2}}+\overline{\zeta^{2}}\right)+\rho \bar{h}\right]=0 . \quad .
$$

Now it is easily seen that $\delta \bar{h} / \delta t$ and $d \bar{h} / d t$ are equivalent, since $\bar{h}$ cannot be affected by external forces any more than by convection; therefore

$$
\begin{aligned}
\frac{1}{2} \rho \frac{d}{d t} \overline{\xi^{2}}+ & \eta^{\overline{2}}+\overline{\zeta^{2}}+\rho \frac{d \bar{h}}{d t}+\rho \overline{\xi^{2}} a+\rho \overline{\eta^{2} b}+\rho \overline{\bar{\zeta}^{2}} c+\rho \overline{\eta \zeta} \mathrm{A}+\rho \overline{\zeta \xi} \mathrm{B}+\rho \overline{\xi \eta} \mathrm{C} \\
& +\frac{1}{2} \frac{\partial}{\partial x}\left(\rho \overline{\xi^{3}}+\rho \overline{\xi \eta^{2}}+\rho \overline{\xi \zeta^{2}}\right)+\frac{1}{2} \frac{\partial}{\partial y}\left(\rho \overline{\eta \xi^{2}}+\rho \overline{\eta^{3}}+\rho \overline{\eta \zeta^{2}}\right) \\
& +\frac{1}{2} \frac{\partial}{\partial z}\left(\rho \overline{\zeta \xi^{2}}+\rho \overline{\zeta \eta^{2}}+\rho \overline{\zeta^{3}}\right)=0 . \quad . . . . . \cdot(31)
\end{aligned}
$$

Maxwell puts $\rho \bar{h}=\frac{1}{2}(\beta-1) \rho\left(\overline{\xi^{2}}+\overline{\eta^{2}}+\overline{\zeta^{2}}\right)$; if this relation is adopted, equation (31). will be that numbered (94) in Maxwell's paper, with a difference of factor, however, in the terms relating to conduction of heat. The foregoing deduction will not be subject to the criticism which M. Poincaré has offered with respect to Maxwell's deduction (see Comptes Rendus, vol. cxvi. p. 1017 ; see also ibidem, p. 1165, where the internal energy of the molecules is taken into account in the same way as that here adopted).

XLVI. Experiments on the Radiation of Heated Gases. $B y$ JoHN Evershed*.

[Plate VII.]

THE renewed interest aroused in certain fundamental 1 questions in spectroscopy by the publication of the researches of Pringsheim, Paschen, and Smithells has led me recently to undertake a series of observations on the visible

* Communicated by the Autbor. 